\title{
Global properties of locally spatially homogeneous cosmological models with matter
}

\author{
Alan D. Rendall* \\ Max-Planck-Institut für Astrophysik \\ Karl-Schwarzschild-Str. 1 \\ Postfach 1523 \\ 85740 Garching \\ Germany
}

\begin{abstract}
The existence and nature of singularities in locally spatially homogeneous solutions of the Einstein equations coupled to various phenomenological matter models is investigated. It is shown that, under certain reasonable assumptions on the matter, there are no singularities in an expanding phase of the evolution and that unless the spacetime is empty a contracting phase always ends in a singularity where at least one scalar invariant of the curvature diverges uniformly. The class of matter models treated includes perfect fluids, mixtures of non-interacting perfect fluids and collisionless matter.
\end{abstract}

\section{Introduction}

When trying to understand the global properties of solutions of the Einstein equations, a tractable starting point is the study of solutions with high symmetry. Assuming that there is a symmetry group with three-dimensional spacelike orbits leads to the class of spatially homogeneous spacetimes. More generally, it is possible to consider spacetimes which are locally isometric to spatially homogeneous ones. For these spacetimes the Einstein equations reduce to ordinary differential equations, an enormous simplification. At the same time these spacetimes are general enough to display a variety of interesting dynamical behaviour.

* Present address: Institut des Hautes Etudes Scientifiques, 35 Route de Chartres, 91440 Bures sur Yvette, France 
The purpose of this paper is to study the global properties of locally spatially homogeneous cosmological models which admit a compact Cauchy surface. Attention will be confined to certain phenomenological matter models. No attempt will be made to determine the details of the evolution of these spacetimes; the aim is rather to answer two fundamental questions. The first is whether singularities can occur in an expanding phase of the evolution of the spacetime and the second is whether a singularity which occurs in a contracting phase must be a curvature singularity. A review of results on these questions in the case where the matter is a perfect fluid can be found in [6].

The class of cosmological models to be considered will now be defined. They all admit Cauchy surfaces and have a matter content which is such that the Cauchy problem for the Einstein equations coupled to the equations describing the matter model is well-posed. Hence it is enough to define the class of initial data which gives rise to them. The initial data consists of a Riemannian metric $g_{a b}$, a symmetric tensor $k_{a b}$ and some matter data, denoted collectively by $F_{0}$, on a three-dimensional manifold $M$.

Definition An initial data set $\left(g_{a b}, k_{a b}, F_{0}\right)$ for the Einstein-matter equations is called locally homogeneous if the naturally associated data set on the universal covering manifold $\tilde{M}$ is homogeneous i.e. invariant under a transitive group action.

The terminology is motivated by the following fact. A Riemannian manifold $(M, g)$ is called locally homogeneous if given any two points $x, y \in M$ there exists an isometry $\phi$ of a neighbourhood of $x$ onto a neighbourhood of $y$ with $\phi(x)=y$. Singer's theorem [14] says that a complete Riemannian manifold $M$ is locally homogeneous if and only if its universal cover is homogeneous. It is left open here whether an analogue of Singer's theorem holds for initial data sets for the Einstein equations. The spacetimes considered in the following will be Cauchy developments of locally homogeneous initial data sets on compact manifolds. It is convenient for many purposes to work on the universal covering space $\tilde{M}$. In the notation no distinction will be made between objects on $M$ and their pull-backs to $\tilde{M}$.

The universal cover of the given spacetime may not be spatially homogeneous but it can be extended to be spatially homogeneous. (This follows from the fact that the equations describing the evolution of the geometry and the matter fields on the universal cover can be written in a form where there is no explicit dependence on the spatial variables.) It will be assumed that such an extension has been made. Then the universal cover has a preferred foliation by orbits. Each leaf of this foliation has constant mean curvature. There is an induced foliation of the original spacetime by constant mean curvature hypersurfaces. It is then topologically of the form $M \times I$, where $I$ is an interval. A time coordinate can be defined by requiring that it be zero on the initial hypersurface and that it be an arc length parameter increasing towards the future on the timelike geodesics normal to the initial hypersurface. This time coordinate is constant on each leaf of the preferred foliation. A time coordinate of this kind will be called Gaussian. Let the mean curvature of the leaf with time coordinate $t$ be denoted by $H(t)$. In the following the time orientation will be chosen so that the mean curvature of the initial hypersurface $t=0$ is non-positive. In other words the model is initially non-contracting.

The spatially homogeneous spacetimes can be divided into two classes, the Bianchi 
models and the Kantowski-Sachs models. (For general information on this see [16].) Most of this paper is concerned with the Bianchi case. The Kantowski-Sachs case is discussed in Section 5. For Bianchi models the universal cover $\tilde{M}$ can be identified with a Lie group $G$. Tensors on $G$ will be described in terms of frame components in a left invariant frame. The components of the induced metric and second fundamental form of the homogeneous hypersurfaces will be denoted by $g_{i j}$ and $k_{i j}$ respectively. They are functions of $t$. The mean curvature is given by $H=g^{i j} k_{i j}$.

The matter models to be considered will be defined in terms of some general properties. As usual $T^{\alpha \beta}$ denotes the energy-momentum tensor. When a specific matter model has been chosen $T^{\alpha \beta}$ will be a functional of some matter variables, denoted collectively by $F$, and the spacetime metric $g_{\alpha \beta}$. In the following another quantity $N^{\alpha}$ (called the particle current density) will be required. It is also assumed to be a functional of $F$ and $g_{\alpha \beta}$. Now various properties which will be assumed at appropriate points will be listed.

(1) $T^{\alpha \beta} V_{\alpha} W_{\beta} \geq 0$ for all future-pointing timelike vectors $V^{\alpha}$ and $W^{\alpha}$ (dominant energy condition)

(2) $T^{\alpha \beta}\left(g_{\alpha \beta}+V_{\alpha} V_{\beta}\right) \geq 0$ for all unit timelike vectors $V^{\alpha}$ (non-negative sum pressures condition)

(3) for any $F$ and $g_{\alpha \beta}$ the conditions $\nabla_{\alpha} N^{\alpha}=0$ and $\nabla_{\alpha} T^{\alpha \beta}=0$ are satisfied (conservation conditions)

(4) for any $F$ and $g_{\alpha \beta}$ the vector $N^{\alpha}$ is future-pointing timelike or zero

(5) for any constant $C_{1}>0$ there exists a positive constant $C_{2}$ such that for any $F$ and $g_{\alpha \beta}$ with $-N_{\alpha} N^{\alpha} \leq C_{1}$ and any timelike vector $V^{\alpha}$ the following inequality holds:

$$
T^{\alpha \beta} V_{\alpha} V_{\beta} \geq C_{2}\left(N^{\alpha} V_{\alpha}\right)^{2}
$$

(6) for any constant $C_{1}>0$ there exists a positive constant $C_{2}<1$ such that for any $F$ and $g_{\alpha \beta}$ with $-N^{\alpha} N_{\alpha} \leq C_{1}$ and any unit timelike vector $V^{\alpha}$

$$
\left(g_{\alpha \beta}+V_{\alpha} V_{\beta}\right) T^{\alpha \beta} \leq 3 C_{2} T^{\alpha \beta} V_{\alpha} V_{\beta}
$$

(7) if a solution with the given symmetry of the Einstein equations coupled to the given matter model is such that the time coordinate defined above takes all values in the interval $\left(t_{1}, t_{2}\right)$, if it is not possible to extend the spacetime so as to make this interval longer and if $t_{1}$ or $t_{2}$ is finite then $H(t)$ is unbounded in a neighbourhood of $t_{1}$ or $t_{2}$ respectively.

(8) for any constant $C_{1}>0$ there exists a constant $C_{2}>0$ such that $T_{\alpha \beta} T^{\alpha \beta} \leq C_{1}$ implies $-N_{\alpha} N^{\alpha} \leq C_{2}$

Some comments will now be made concerning the physical motivation of some of these conditions. If a given type of matter can be considered as being made up of particles then 
a particle current density $N^{\alpha}$ is defined. If the particles have positive rest mass then this vector is future pointing timelike or zero as required by condition (4). If the particles are massless then this condition is still satisfied except for very special types of matter where $N^{\alpha}$ might be null. If particles cannot be created or destroyed then $N^{\alpha}$ is divergenceless as required in condition (3). It is not easy to give an intuitive interpretation of conditions (5) and (6). The meaning of (5) is roughly as follows. If matter is observed from a boosted frame then the particle density is multiplied by a $\gamma$-factor arising from the effect of Lorentz contraction on the volume element. The observed energy density is also affected in this way but picks up an additional $\gamma$-factor. Hence when a given matter distribution is considered from a boosted frame the multiplicative factor in the observed energy density behaves like the square of that in the observed particle density. As for condition (6), it will be seen in Section 4 that for a perfect fluid it is related to the condition that the speed of sound should be bounded away from the speed of light. The given symmetry referred to in condition (7) will be Bianchi symmetry or Kantowski-Sachs symmetry, according to the context.

In the following the two fundamental questions formulated above will be answered for locally spatially homogeneous spacetimes which are spatially compact and where the matter content satisfies (some of) the conditions (1)-(8). In fact it will also be necessary when examining the issue of curvature singularities to assume that the spacetimes considered are not empty since in the vacuum case it is much more difficult to separate spacetimes with curvature singularities from spacetimes with Cauchy horizons. The question of which spatially compact, locally spatially homogeneous vacuum spacetimes have Cauchy horizons has been investigated in [3]. In the following charged matter and field-theoretic matter models (e.g. Yang-Mills, $\sigma$-models) are not considered. It would be of interest to know whether similar results hold in those cases.

The paper is organized as follows. Section 2 contains results on geodesic completeness in an expanding phase while Section 3 is concerned with curvature singularities in a contracting phase. In both cases the hypotheses on the matter model are of a general nature. Section 4 is concerned with verifying that these properties hold for various specific matter models, namely perfect fluids, non-interacting mixtures of perfect fluids and collisionless matter. As already mentioned, the last section is devoted to Kantowski-Sachs models.

\section{Geodesic completeness}

In this section it will be shown that under general conditions there can be no singularity during an expanding phase of a locally spatially homogeneous spacetime. For this statement the existence of a compact Cauchy surface is irrelevant. An expanding phase means by definition a time interval $\left(t_{1}, t_{2}\right)$ where $H<0$. The statement that there are no singularities means that either the solution can be extended to an interval $\left(t_{1}, t_{2}^{\prime}\right)$ with $t_{2}^{\prime}>t_{2}$ or that it is future complete (i.e. that all inextendible causal geodesics are complete in the future direction). This statement is a consequence of the following theorem.

Theorem 2.1 Let a spatially locally homogeneous solution of the Einstein equations coupled to a matter model satisfying the conditions (1), (2) and (7) be given. Suppose that a Gaussian time coordinate defined on this spacetime takes all values in the interval $\left(t_{1}, t_{2}\right)$ and that the spacetime cannot be extended so as to enlarge this interval. Suppose 
further that the mean curvature $H$ satisfies $\lim \sup _{t \rightarrow t_{2}} H(t)<\infty$. Then $t_{2}=\infty$ and the spacetime is future complete.

Proof The proof given here is for Bianchi models; for Kantowski-Sachs models see Section 5. Since spatial compactness plays no role we may assume without loss of generality that the spacetime is simply connected. Now some of the Einstein equations for Bianchi models are needed, namely the Hamiltonian constraint

$$
R-k_{i j} k^{i j}+H^{2}=16 \pi T^{00}
$$

and the evolution equation

$$
\partial_{t} H=R+H^{2}-12 \pi T^{00}+4 \pi g_{i j} T^{i j} .
$$

Here $R$ denotes the scalar curvature of the spatial metric. Combining these two equations gives

$$
\partial_{t} H=k_{i j} k^{i j}+4 \pi\left(T^{00}+g_{i j} T^{i j}\right) .
$$

Now $k_{i j} k^{i j} \geq \frac{1}{3} H^{2}$ and so equation (2.3) together with conditions (1) and (2) implies that $\partial_{t} H \geq \frac{1}{3} H^{2}$. In particular this shows that $H$ is non-decreasing. Thus, under the hypotheses of the theorem, the fact that $t_{2}=\infty$ follows immediately from condition (7). The inequality also shows that if $H$ is ever positive it must become infinite in finite time. Since it is already known that $t_{2}=\infty$ it follows that $H \leq 0$. There are now two possibilities: either $H$ vanishes at some time or it is everywhere negative.

Suppose that $H=0$ at some time $t_{0}$. Since $H$ is everywhere non-positive it can be concluded that $\partial_{t} H$ is also zero there. From equation (2.3) it follows that $k_{i j}=0$ and $T^{00}=0$ for $t=t_{0}$. The dominant energy condition implies that $T^{\alpha \beta}=0$ at $t=t_{0}$ and hence (see [10], p. 94) everywhere. Thus in this case the solution must be a vacuum solution. Under these conditions the Einstein evolution equations imply that the Ricci tensor $R_{i j}$ of $g_{i j}$ is zero at $t_{0}$. Hence the metric $g_{i j}\left(t_{0}\right)$ is flat. Uniqueness in the Cauchy problem for the vacuum Einstein equations now shows that in the first case the spacetime is obtained by an identification of (the whole of) Minkowski space and future completeness is clear.

Now consider the second case. There the universe expands for ever. It has been shown by Lin and Wald [11] that for Bianchi IX models conditions (1) and (2) imply that the universe cannot expand for ever. Thus under the hypotheses of the theorem the Bianchi type cannot be IX. It can then be concluded that $R \leq 0$. (For a simple proof of this see [15].) Using this in (2.1) gives

$$
k_{i j} k^{i j} \leq H^{2}
$$

The eigenvalues of $k_{i j}$ with respect to $g_{i j}$ are the solutions of

$$
\operatorname{det}\left(k_{i j}-\lambda g_{i j}\right)=0
$$

Call them $\lambda_{1}, \lambda_{2}$ and $\lambda_{3}$. Then

$$
\lambda_{1}+\lambda_{2}+\lambda_{3}=H,
$$


and

$$
\lambda_{1}^{2}+\lambda_{2}^{2}+\lambda_{3}^{2}=k_{i j} k^{i j}
$$

Define $p_{i}=\lambda_{i} / H$ so that

$$
\begin{gathered}
p_{1}+p_{2}+p_{3}=1 \\
p_{1}^{2}+p_{2}^{2}+p_{3}^{2} \leq 1
\end{gathered}
$$

Let $K$ denote the subset of $\mathbf{R}^{3}$ where the relations (2.8) are satisfied. The minimum value of $p_{1}$ on $K$ will now be determined. By symmetry this will also be the minimum value of $p_{2}$ and $p_{3}$. The minimum must occur on the boundary of $K$ and the boundary is given by replacing the inequality in $(2.8)$ by equality. Since $K$ is convex the minimum must occur when two of the eigenvalues are equal and using this its value can be computed to be $-1 / 3$. It follows that for any vector $x^{i}$ tangent to the hypersurfaces of homogeneity the following inequality holds

$$
k_{i j} x^{i} x^{j} \leq-\frac{1}{3} H g_{i j} x^{i} x^{j} .
$$

Consider now a future directed causal geodesic and let $q^{i}$ be the frame components of the projection of its tangent vector onto the hypersurfaces of constant $t$. Then

$$
\begin{aligned}
\frac{d}{d t}\left(g_{i j} q^{i} q^{j}\right) & =2 k_{i j} q^{i} q^{j} \\
& \leq-\frac{2}{3} H g_{i j} q^{i} q^{j}
\end{aligned}
$$

Using the inequality $\partial_{t} H \geq \frac{1}{3} H^{2}$ once more shows that

$$
H(t) \geq-3 /(C+t)
$$

for some positive constant $C$. Combining (2.10) and (2.11) gives

$$
\frac{d}{d t}\left(\log g_{i j} q^{i} q^{j}\right) \leq 2 /(C+t)
$$

Hence

$$
\left(g_{i j} q^{i} q^{j}\right)^{-1 / 2} \geq C(1+t)^{-1}
$$

The affine parameter length of the geodesic up to a certain point is given by the integral of $\left(\epsilon+g_{i j} q^{i} q^{j}\right)^{-1 / 2}$ with respect to $t$, where $\epsilon$ is 1 for a timelike geodesic parametrized by arc length and zero for a null geodesic. Future geodesic completeness is equivalent to the divergence of this integral as the upper limit tends to infinity. This is guaranteed by $(2.13)$.

\section{Curvature singularities}

The subject of this section is a contracting phase of the universe i.e. a time interval $\left(t_{1}, t_{2}\right)$ where $H>0$. Only Bianchi models are considered here - analogous results for spacetimes with Kantowski-Sachs symmetry are presented in Section 5. It was shown in 
the last section that conditions (1) and (2) imply that $H$ is non-decreasing. It follows that any extension of the solution to an interval $\left(t_{1}, t_{2}^{\prime}\right)$ with $t_{2}^{\prime}>t_{2}$ must also be a contracting phase in this sense. Extend the time interval as far as possible to the future and call the result $\left(t_{1}, t_{*}\right)$. It was also remarked in the last section that if $H$ is positive somewhere it must become infinite in finite time. Hence $t_{*}<\infty$. In this section, in contrast to the previous section, the spatial compactness of the model plays an important role. Also all the conditions (1)-(7) will be used. Writing out the equation $\nabla_{\alpha} N^{\alpha}=0$ explicitly for a Bianchi model gives

$$
\partial_{t}\left((\operatorname{det} g)^{1 / 2} N^{0}\right)=-C_{i j}^{i} N^{j}(\operatorname{det} g)^{1 / 2}
$$

Here $C_{j k}^{i}$ are the structure constants of the Lie algebra corresponding to the Lie group being considered. This equation should be thought of as being defined on the universal covering spacetime where an invariant frame exists. In the Bianchi types of class A where, by definition, $C_{i j}^{i}=0$ the right hand side of (3.1) vanishes identically and the quantity $(\operatorname{det} g)^{1 / 2} N^{0}$ is conserved. It will now be shown that the assumption of spatial compactness is enough to guarantee the vanishing of the right hand side of (3.1), even for class B models. To see this let $X$ be any vector on the Lie group $G$ which is left invariant and which projects to a smooth vector field $\bar{X}$ on $M$. The divergence of $X$ is constant because it is invariant and the divergence of $\bar{X}$ is the same constant. However the integral of $\operatorname{div} \bar{X}$ over $M$ must be zero by Stokes' theorem. It follows that $X$ has divergence zero. In terms of components this divergence is just $C_{i j}^{i} X^{j}$. Now $N^{j}$ is at each time a vector field whose pull-back to the universal covering manifold has the properties of the vector field $X$ just discussed. It follows that the right hand side of (3.1) is zero as claimed.

The aim now is to show that if conditions (1)-(7) hold and if $N^{\alpha}$ does not vanish then the singularity which occurs as $t \rightarrow t_{*}$ is a curvature singularity. Because of the conserved quantity which has been found it suffices to assume that $N^{\alpha}$ does not vanish on the initial hypersurface to ensure that it does not vanish anywhere.

Theorem 3.1 Let a solution with Bianchi symmetry of the Einstein equations coupled to a matter model satisfying conditions (1)-(7) and admitting a compact Cauchy hypersurface be given. Suppose that a Gaussian time coordinate defined on this spacetime takes all values in the interval $\left(t_{1}, t_{*}\right)$ and that the spacetime cannot be extended so as to enlarge this interval. Suppose further that $H$ is positive somewhere and that the particle current density $N^{\alpha}$ does not vanish. Then the mass density measured by any observer is unbounded as $t \rightarrow t_{*}$. If in addition condition (8) is satisfied then $\lim \sup _{t \rightarrow t_{*}} G^{\alpha \beta} G_{\alpha \beta}(t)=\infty$, where $G_{\alpha \beta}$ is the Einstein tensor of $g_{\alpha \beta}$.

Proof The first thing to be done is to obtain some control on the rate at which $H(t)$ blows up as $t \rightarrow t_{*}$. (That it does blow up as $t \rightarrow t_{*}$ follows from condition (7).) It was already mentioned in the previous section that $\partial_{t} H \geq \frac{1}{3} H^{2}$. Integrating this inequality gives

$$
H(t) \leq \frac{3}{t_{*}-t}
$$

On the other hand (2.2) and the dominant energy condition imply that

$$
\partial_{t} H \leq R+H^{2}
$$


Let $R_{+}(t)=\max (R(t), 0)$. Then $\partial_{t} H \leq R_{+}+H^{2}$. For $t$ close to $t_{*}$ we have $H \geq 1$. Thus $\partial_{t} H \leq R_{+} H+H^{2}$ and integrating this inequality gives the estimate

$$
H(t) \geq\left(t_{*}-t\right)^{-1} \exp \left(-\int_{t}^{t_{*}} R_{+}\left(t^{\prime}\right) d t^{\prime}\right)
$$

Here the right hand side is defined to be zero if the integral is infinite. If the Bianchi type is not IX then $R_{+}=0$ and (3.4) simplifies to

$$
H(t) \geq\left(t_{*}-t\right)^{-1}
$$

From these estimates for $H$ it is possible to get estimates for $\operatorname{det} g$ since $d / d t(\log \operatorname{det} g)=$ $-2 H$. It follows from (3.2) that

$$
\operatorname{det} g \geq C_{1}\left(t_{*}-t\right)^{6}
$$

for some positive constant $C_{1}$. If $\int_{t}^{t_{*}} R_{+}(t) d t<\infty$ for any $t<t_{*}$ then (3.4) implies that

$$
\operatorname{det} g(t) \leq C_{2}\left(t_{*}-t\right)^{2}
$$

The integrability condition on the scalar curvature is obviously satisfied when the Bianchi type is not IX. In type IX a more detailed analysis is necessary to see whether it holds. When (3.7) holds the fact that $N^{0}(\operatorname{det} g)^{1 / 2}$ is conserved shows that

$$
N^{0} \geq C_{3}\left(t_{*}-t\right)^{-1}
$$

Applying condition (5) to the unit normal vector to the homogeneous hypersurfaces gives

$$
T^{00} \geq C N_{0}^{2}
$$

as long as $N_{\alpha} N^{\alpha}$ is bounded.

Suppose now that the Bianchi type is not IX. It will be shown that the assumption that $N_{\alpha} N^{\alpha}$ is bounded leads to a contradiction in this case. If this quantity is bounded then (3.8) and (3.9) imply that there exists a constant $C>0$ such that

$$
T^{00} \geq C\left(t_{*}-t\right)^{-2}
$$

Then the Hamiltonian constraint (2.1) and the fact that $R \leq 0$ when the Bianchi type is not IX give an estimate of the form

$$
k_{i j} k^{i j} \leq H^{2}(1-\eta), \eta>0
$$

Taking a linear combination of (2.1) and (2.2) gives

$$
\partial_{t} H=\alpha\left(k_{i j} k^{i j}-H^{2}\right)+(1-\alpha) R+H^{2}-4 \pi(3-4 \alpha) T^{00}+4 \pi g_{i j} T^{i j}
$$


Because of (6) it is possible to choose $\alpha>0$ so that the sum of the last two terms in this inequality is not positive. Hence

$$
\partial_{t} H \leq(1-\alpha \eta) H^{2}
$$

This allows the inequality (3.8) to be improved to

$$
N^{0} \geq C\left(t_{*}-t\right)^{\frac{-1}{1-\alpha \eta}}
$$

From (3.9) it follows that

$$
T^{00} \geq C\left(t_{*}-t\right)^{\frac{-2}{1-\alpha \eta}}
$$

This contradicts the Hamiltonian constraint. The conclusion is that if the Bianchi type is not IX then $N^{\alpha} N_{\alpha}$ must be unbounded. Consider now any observer, represented mathematically by a timelike curve with unit tangent vector $t^{\alpha}$. Then the mass density measured by this observer is

$$
-N^{\alpha} t_{\alpha} \geq \sqrt{-N_{\alpha} N^{\alpha}}
$$

This gives the first conclusion of the theorem in the case that the Bianchi type is not IX. The other conclusion follows immediately from the fact that $N^{\alpha} N_{\alpha}$ is unbounded.

Consider now the Bianchi IX case. It is a standard fact (see e.g. [15]) that the dimensionless quantity $(\operatorname{det} g)^{1 / 3} R$ is bounded above. Hence $R \leq C(\operatorname{det} g)^{-1 / 3}$. Suppose for a moment that

$$
\operatorname{det} g \geq C\left(t_{*}-t\right)
$$

for some positive constant $C$. Then $R_{+} \leq C\left(t_{*}-t\right)^{-1 / 3}$. This implies the integrability of $R_{+}$and hence, by (3.4), that $H(t) \geq C /\left(t_{*}-t\right)$. It follows that $\operatorname{det} g$ tends to zero as $t \rightarrow t_{*}$. If, on the other hand, the inequality (3.17) does not hold for any constant $C$ then there must exist a sequence of times $t_{n}$ with $t_{n} \rightarrow t_{*}$ such that

$$
\operatorname{det} g\left(t_{n}\right) \leq C\left(t_{*}-t_{n}\right)
$$

Hence, in particular, $\operatorname{det} g\left(t_{n}\right) \rightarrow 0$. Since $\operatorname{det} g$ is monotonically decreasing near the singularity it follows that $\operatorname{det} g \rightarrow 0$ in this case too.

It will now be shown that the assumption that $N_{\alpha} N^{\alpha}$ is bounded leads to a contradiction. This suffices to complete the proof of the theorem since from that point on the argument is identical to that given above for the case where the Bianchi type is not IX. In the equation (2.2) the combination $R-12 \pi T^{00}+4 \pi g_{i j} T^{i j}$ occurs. If $N^{\alpha} N_{\alpha}$ is bounded then condition (6) allows the sum of the second and third terms to be bounded from above by $-C T^{00}$ for some positive constant $C$. Also condition (5) gives

$$
T^{00} \geq C\left(N^{0}\right)^{2}=C^{\prime}(\operatorname{det} g)^{-1}
$$

Thus as det $g$ tends to zero $C T^{00}$ becomes larger than $R$ and the above combination of terms from $(2.2)$ is negative. Hence $\partial_{t} H \leq H^{2}$. It follows that the inequalities (3.7), (3.8) and (3.10) remain true in the Bianchi IX case. The inequality (3.11) can also be obtained if 
we replace the fact that $R \leq 0$ used before by the fact that sufficiently near the singularity $R \leq C T^{00}$ for any given positive constant $C$. This can also be used to control the term involving $R$ in (3.12) using a small constant multiplied by $T^{00}$. This is enough to prove (3.13)-(3.15) in the Bianchi IX case. The same trick of dominating $R$ by a small amount of $T^{00}$ shows that (3.15) contradicts the Hamiltonian constraint in this case also and this completes the proof of the theorem.

Remarks 1. When applied to a general perfect fluid solution of Bianchi type IX this theorem is a non-trivial generalization of the results given in [6].

2. In [12] it was claimed that a singularity in a contracting phase of a Bianchi class A nonvacuum solution of the Vlasov-Einstein system must be a curvature singularity. The claim is not justified by the arguments given there but it is true. It follows from Theorem 3.1 above and the fact, proved in the next section, that the Vlasov equation satisfies conditions (1)-(8).

3. For solutions where $N^{\alpha}$ is orthogonal to the hypersurfaces $t=$ const. $\sqrt{-N^{\alpha} N_{\alpha}}=N^{0}$ and so in that case the theorem remains true if conditions (5) and (6) are dropped from the assumptions.

\section{Matter models}

Before specific matter models are studied some general remarks will be made concerning condition (7) in spacetimes with Bianchi symmetry. Suppose that a solution is given on an interval $\left(t_{1}, t_{2}\right)$, that $t_{2}$ is finite and that $H$ is bounded on a neighbourhood of $t_{2}$. Then det $g$ and its inverse are bounded on a neighbourhood of $t_{2}$. It is a standard fact for Bianchi models that under these conditions the scalar curvature $R$ is bounded above (see e. g. [15]). Next the Hamiltonian constraint shows that $k^{i j} k_{i j}$ is bounded. An argument given in [12] (p. 88-89) then shows that $g_{i j}$ and $k_{i j}$ are bounded in a neighbourhood of $t_{2}$. Analogous statements hold in the case that the roles of $t_{1}$ and $t_{2}$ are interchanged. What is needed to check condition (7) is that when a solution of the Einstein-matter equations is given on some open interval and when $g_{i j}, k_{i j}$ and $(\operatorname{det} g)^{-1}$ are bounded near an endpoint of this interval, then the solution extends through this endpoint to a strictly longer interval. Call this condition $\left(7^{\prime}\right)$.

It is now time to look at some examples. The matter model which has been studied most extensively in the context of Bianchi models is the perfect fluid. It will now be investigated under which conditions on the equation of state a perfect fluid satisfies conditions (1)-(8). The equation of state is a relation $p=f(\rho)$ between energy density and pressure. The energy-momentum tensor is $T^{\alpha \beta}=\rho u^{\alpha} u^{\beta}+(\rho+p) g^{\alpha \beta}$ The equation of state will be assumed to satisfy the following conditions

(i) $f$ is a continuous function from $[0, \infty)$ to the non-negative real numbers with $f(0)=0$ which is $C^{1}$ for $\rho>0$

(ii) $0 \leq d f / d \rho \leq 1$ for each $\rho>0$

Condition (1) is satisfied if $|p| \leq \rho([10]$, p. 91) while condition (2) is equivalent to $p \geq 0$. Hence both of these conditions are consequences of (i) and (ii). The quantity $N^{\alpha}$ 
is defined to be $r u^{\alpha}$, where

$$
r=\exp \left\{\int_{1}^{\rho}\left(\rho^{\prime}+f\left(\rho^{\prime}\right)\right)^{-1} d \rho^{\prime}\right\}
$$

This definition ensures that the Euler equations $\nabla_{\alpha} T^{\alpha \beta}=0$ imply $\nabla_{\alpha} N^{\alpha}=0$ and hence that (3) is satisfied. Condition (4) obviously holds. A sufficient condition for (5) to be true is that an upper bound on $r$ implies an inequality of the form $\rho \geq C r^{2}$. In fact this follows from (4.1) and the dominant energy condition. Condition (6) represents a restriction on the equation of state which goes beyond (i) and (ii), namely that when $\rho$ is less than some given constant $C_{1}$ there is a constant $C_{2}<1$ such that $p \leq C_{2} \rho$. It will be expressed by saying that the fluid is not asymptotically stiff at low densities. Condition (8) obviously holds.

The one condition which remains to be verified for a perfect fluid is $\left(7^{\prime}\right)$. In order to do this it is useful to write the Euler equation $\nabla_{\alpha} T^{\alpha \beta}=0$ in terms of the density $\rho$ and the spatial components $v^{i}$ of the four-velocity (expressed in an invariant frame). Then no spatial derivatives occur and a system of ordinary differential equations is obtained. If we denote the matter variables $\rho$ and $u^{i}$ collectively by $f$ then the equations take the form

$$
A\left(f, g_{i j}\right) d f / d t=F\left(f, g_{i j}, k_{i j}\right)
$$

where the matrix $A$ and the vector $F$ depend smoothly on their arguments. As long as $\rho>0$ the determinant of $A$ is non-vanishing. Hence the evolution equation can be written as $d f / d t=A^{-1} F$. The right hand side of this latter equation is regular so long as $\operatorname{det} g$ and $\rho$ are bounded away from zero. This equation can be combined with the evolution equations for the geometry, which in the homogeneous case are

$$
\begin{aligned}
& \partial_{t} g_{i j}=-2 k_{i j} \\
& \partial_{t} k_{i j}=R_{i j}+\left(g^{l m} k_{l m}\right) k_{i j}-2 k_{i l} k_{j}^{l}-8 \pi T_{i j}-4 \pi T^{00} g_{i j}+4 \pi\left(g_{l m} T^{l m}\right) g_{i j}
\end{aligned}
$$

The Ricci tensor $R_{i j}$ is a rational function of $g_{i j}$ whose exact form depends on the Bianchi type. Taking the equation for $d f / d t$ together with (4.3) and (4.4) gives a system of ordinary differential equations for $f, g_{i j}$ and $k_{i j}$. The coefficients in these equations are regular as long as $\operatorname{det} g$ and $\rho$ are strictly positive. Standard results on existence and uniqueness for ordinary differential equations (see e.g. [9]) show that given initial data $\left(\rho, u^{i}, g_{i j}, k_{i j}\right)$ with $\rho>0$ there exists a unique solution of these equations on some time interval with the given initial values. Moreover this solution can be extended as long as $u^{i}, g_{i j}, k_{i j}, \rho, \rho^{-1}$ and $(\operatorname{det} g)^{-1}$ remain bounded.

To verify condition $\left(7^{\prime}\right)$ it remains to show that if a solution of the Einstein-Euler system is given on some time interval and if $g_{i j}, k_{i j}$ and $(\operatorname{det} g)^{-1}$ are bounded then the relevant matter quantities are bounded. The conservation law for $N^{0}$ shows that $r u^{0}$ and its inverse are bounded. Hence it can be concluded that $r$ is bounded above. For a perfect fluid condition (6) implies that for $r \leq C_{1}$ an inequality of the form $\rho \geq C_{2} r^{2-\epsilon}$ holds, for some $\epsilon>0$. Now

$$
\begin{aligned}
T^{00} & \geq \rho\left(u^{0}\right)^{2} \geq C r^{2-\epsilon}\left(u^{0}\right)^{2} \\
& =C\left(r u^{0}\right)^{2} r^{-\epsilon}
\end{aligned}
$$


The Hamiltonian constraint shows that $T^{00}$ is bounded and so it follows that $r^{-1}$ is bounded. Combining this with the boundedness of $r u^{0}$ shows that $u^{0}$ is bounded. This completes the verification of condition $\left(7^{\prime}\right)$ for a perfect fluid.

The above information concerning perfect fluids will now be collected.

Proposition 4.1 Consider a perfect fluid with equation of state $p=f(\rho)$ satisfying conditions (i) and (ii). Then conditions (1)-(5)and (8) are satisfied. If, in addition, the fluid is not asymptotically stiff at low densities then conditions (6) and (7) are also satisfied.

It follows from this proposition that Theorem 3.1 applies to any perfect fluid which satisfies the conditions (i) and (ii) and which is not asymptotically stiff at low densities.

Another kind of matter model which has been popular in cosmology is that of $n$ (usually two) non-interacting perfect fluids [4]. The energy-momentum tensor in this case is of the form

$$
T^{\alpha \beta}=T_{(1)}^{\alpha \beta}+\ldots+T_{(n)}^{\alpha \beta}
$$

where each summand $T_{(i)}^{\alpha \beta}$ has the form of the energy-momentum tensor of a perfect fluid with its own four-velocity $u_{(i)}^{\alpha}$, density $\rho_{(i)}$ and pressure $p_{(i)}$. The latter are related by equations of state $p_{(i)}=f_{(i)}\left(\rho_{(i)}\right)$. The condition that the fluids are non-interacting is expressed by the equations $\nabla_{\alpha} T_{(i)}^{\alpha \beta}=0$ for each $i$. If we define $r_{(i)}$ by putting an index $i$ on all the quantities in (4.1) and let $N_{(i)}^{\alpha}=r_{(i)} u_{(i)}^{\alpha}$ then it follows that $\nabla_{\alpha} N_{(i)}^{\alpha}=0$ for each $i$. In particular the sum $N^{\alpha}$ of the $N_{(i)}^{\alpha}$ has zero divergence. Before showing under which assumptions a mixture of perfect fluids satisfies conditions (1)-(8), a more general result will be given on matter models which are composites of two others.

Lemma 4.1 Let two matter models be given with matter variables $F_{(1)}$ and $F_{(2)}$, particle current densities $N_{(1)}^{\alpha}$ and $N_{(2)}^{\alpha}$ and energy-momentum tensors $T_{(1)}^{\alpha \beta}$ and $T_{(2)}^{\alpha \beta}$. Define a new (composite) matter model by taking as matter variables $F=\left(F_{(1)}, F_{(2)}\right)$ and defining $N^{\alpha}$ and $T^{\alpha \beta}$ as the sums of the corresponding quantities for the individual matter models. If the original matter models satisfy conditions (1)-(6) then the composite model does so too.

Proof It is obvious that the composite matter model satisfies conditions (1)-(4). Next note that $-N^{\alpha} N_{\alpha} \geq-N_{(1)}^{\alpha} N_{\alpha}^{(1)}-N_{(2)}^{\alpha} N_{\alpha}^{(2)}$, so that the boundedness of $N^{\alpha} N_{\alpha}$ implies that of $N_{(i)}^{\alpha} N_{\alpha}^{(i)}$ for $i=1,2$. It follows that condition (6) is satisfied and condition (5) is a consequence of the following computation:

$$
\begin{aligned}
T_{\alpha \beta} V^{\alpha} V^{\beta} & =T_{\alpha \beta}^{(1)} V^{\alpha} V^{\beta}+T_{\alpha \beta}^{(2)} V^{\alpha} V^{\beta} \\
& \geq C_{2}\left[\left(N_{\alpha}^{(1)} V^{\alpha}\right)^{2}+\left(N_{\alpha}^{(2)} V^{\alpha}\right)^{2}\right] \\
& \geq \frac{1}{2} C_{2}\left(N^{\alpha} V_{\alpha}\right)^{2}
\end{aligned}
$$

Consider now a non-interacting mixture of perfect fluids and suppose that each individual fluid satisfies the hypotheses of Proposition 4.1 including the condition that it should not be asymptotically stiff. It then follows from Lemma 4.1 that the mixture satisfies conditions (1)-(6). It will now be investigated whether conditions (7) and (8) also hold. The evolution 
equations for a mixture of non-interacting fluids can be written in a similar way to those for a single fluid. Thus to verify condition (7) in this case it suffices to check that the fluid quantities remain finite in a given regular spacetime. However each fluid satisfies the Euler equations separately and so the argument given above for a single fluid applies without change. (It must, of course, be assumed for this that none of the fluids are asymptotically stiff at low densities.) A straightforward calculation shows that

$$
T^{\alpha \beta} T_{\alpha \beta} \geq\left(\sum_{(i)} \rho_{(i)}\right)^{2}
$$

Hence if $T^{\alpha \beta} T_{\alpha \beta}$ is bounded all the $\rho_{(i)}$ are bounded. Moreover, the boundedness of $T^{\alpha \beta} T_{\alpha \beta}$ implies that the quantities $\rho_{(i)} \rho_{(j)}\left(u_{(i)}^{\alpha} u_{\alpha}^{(j)}\right)^{2}$ are bounded for each $i, j$. It follows that $N_{(i)}^{\alpha} N_{\alpha}^{(j)}$ is bounded for each $i, j$. Thus condition (8) is also satisfied and the following analogue of Proposition 4.1 is obtained.

Proposition 4.2 Consider a mixture of non-interacting perfect fluids, each of which satisfies the hypotheses of Proposition 4.1 including the condition that none of them be asymptotically stiff at low densities. Then conditions (1)-(8) are satisfied.

The next matter model which will be considered is a collisionless gas described by the Vlasov equation. For an introduction to the Vlasov-Einstein system see [7]. Some general information on spatially homogeneous solutions can be found in [12]. In the following we consider a gas of particles of unit mass. The distribution function $f$ is supposed to be a $C^{1}$ function with compact support in velocity space initially and these properties are preserved by the time evolution. The quantities $N^{\alpha}$ and $T^{\alpha \beta}$ are defined in the usual way as certain weighted integrals of $f$. Conditions (1)-(4) are standard properties of solutions of the Vlasov-Einstein system. Condition (7) for this matter model is the statement of Lemma 2.2 of [12]. It remains to check conditions (5), (6) and (8) and to do this we may choose a frame whose timelike member is $V^{\alpha}$ in order to do the calculation. Then the inequalities of (5) and (6) become $\hat{T}^{00} \geq C_{2}\left(\hat{N}^{0}\right)^{2}$ and $\delta^{i j} \hat{T}_{i j} \leq 3 C_{2} \hat{T}^{00}$. (The hats here indicate the use of indices associated to an orthonormal frame.)

$$
\begin{aligned}
-N_{\alpha} N^{\alpha} & =-\left(\int f\left(p^{a}\right) p_{\alpha} / p^{0} d p^{1} d p^{2} d p^{3}\right)\left(\int f\left(q^{a}\right) q^{\alpha} / q^{0} d q^{1} d q^{2} d q^{3}\right) \\
& =-\iint f\left(p^{a}\right) f\left(q^{a}\right) p^{\alpha} q_{\alpha} /\left(p^{0} q^{0}\right) d p^{1} d p^{2} d p^{3} d q^{1} d q^{2} d q^{3} \\
& \geq \iint f\left(p^{a}\right) f\left(q^{a}\right) /\left(p^{0} q^{0}\right) d p^{1} d p^{2} d p^{3} d q^{1} d q^{2} d q^{3} \\
& =\left(\int f\left(p^{a}\right) / p^{0} d p^{1} d p^{2} d p^{3}\right)^{2}
\end{aligned}
$$

Hence

$$
\begin{aligned}
N^{0} & =\int f\left(p^{a}\right) d p^{1} d p^{2} d p^{3} \\
& \leq\left(\int f\left(p^{a}\right) p^{0} d p^{1} d p^{2} d p^{3}\right)^{1 / 2}\left(\int f\left(p^{a}\right) / p^{0} d p^{1} d p^{2} d p^{3}\right)^{1 / 2} \\
& \leq\left(T^{00}\right)^{1 / 2}\left(-N_{\alpha} N^{\alpha}\right)^{1 / 4}
\end{aligned}
$$


This shows that (5) holds. It follows directly from the definitions that the inequality of (6) holds with $C_{2}=1 / 3$ even without restricting $N_{\alpha} N^{\alpha}$ to be bounded. Finally

$$
\begin{aligned}
T_{\alpha \beta} T^{\alpha \beta} & =\left(\int f\left(p^{a}\right) p_{\alpha} p_{\beta} / p^{0} d p^{1} d p^{2} d p^{3}\right)\left(\int f\left(q^{a}\right) q^{\alpha} q^{\beta} / q^{0} d q^{1} d q^{2} d q^{3}\right) \\
& =\iint f\left(p^{a}\right) f\left(q^{a}\right)\left(p^{\alpha} q_{\alpha}\right)^{2} /\left(p^{0} q^{0}\right) d p^{1} d p^{2} d p^{3} d q^{1} d q^{2} d q^{3} \\
& \geq-\iint f\left(p^{a}\right) f\left(q^{a}\right)\left(p^{\alpha} q_{\alpha}\right) /\left(p^{0} q^{0}\right) d p^{1} d p^{2} d p^{3} d q^{1} d q^{2} d q^{3} \\
& =-N_{\alpha} N^{\alpha}
\end{aligned}
$$

Proposition 4.3 Consider a collisionless gas of particles of unit mass for which the distribution function is $C^{1}$ and compactly supported in velocity space. Then conditions (1)-(8) are satisfied.

Most of what was said in the last paragraph only used the fact that matter is described by a distribution function and the expression for the energy-momentum tensor in terms of this distribution function. Hence it has more general applicability to matter models based on kinetic theory. Consider the case of the Boltzmann equation. It follows from the above that conditions (1)-(6) and (8) are satisfied for the Boltzmann equation. The one condition which causes difficulty is (7). Recall furthermore that to verify (7) it is enough to verify $\left(7^{\prime}\right)$. For the Boltzmann equation this is a non-trivial task. Even in the case of spatially homogeneous solutions of the classical Boltzmann equation it is difficult to prove global existence and uniqueness theorems. (For details see [2] and references therein.) Just how difficult it is depends on the form of the collision kernel. For the special relativistic Boltzmann equation there appear to be no results in the literature on global existence of unique solutions for general homogeneous initial data. (There is a result for inhomogeneous data close to equilibrium [8].)

\section{Kantowski-Sachs spacetimes}

In this section a contracting phase of the universe will be considered as in Section 3 but now the symmetry is of Kantowski-Sachs type rather than Bianchi type. It should be mentioned that for this symmetry type no singularities occur in an expanding phase if conditions (1), (2) and (7) hold. It can be shown as in the Bianchi case that if (7) holds either the solution can be extended to the future beyond the expanding phase or the solution exists globally in a Gaussian time coordinate and expands for ever. The impossibility of the second alternative under conditions (1) and (2) has been shown by Burnett[1].

Suppose now that a solution is defined on an interval $\left(t_{1}, t_{*}\right)$ which has been maximally

extended towards the future. Suppose further that this is a contracting phase. In a Kantowski-Sachs spacetime the metric can be written in the form

$$
d s^{2}=-d t^{2}+A^{2}(t) d x^{2}+B^{2}(t) d \Sigma^{2}
$$


where $d \Sigma^{2}$ is the standard metric on the two-sphere. It is easy to check that the divergence of the second fundamental form of the hypersurfaces $t=$ const. is zero. It follows from the momentum constraint that $\partial / \partial t$ is an eigenvector of the energy-momentum tensor with eigenvalue $T^{00}$. Hence

$$
T^{\alpha \beta} T_{\alpha \beta} \geq\left(T^{00}\right)^{2}
$$

It can be shown that, as in the case of the Bianchi models, $(\operatorname{det} g)^{1 / 2} N^{0}$ is time independent. In this case det $g$ denotes the determinant of the three-dimensional metric in the above coordinates, so that $(\operatorname{det} g)^{1 / 2}=A B^{2}$. The following theorem is an analogue of Theorem 3.1 for spacetimes with Kantowski-Sachs symmetry.

Theorem 5.1 Let a solution with Kantowski-Sachs symmetry of the Einstein equations coupled to a matter model satisfying conditions (1)-(7) and admitting a compact Cauchy hypersurface be given. Suppose that a Gaussian time coordinate defined on this spacetime takes all values in the interval $\left(t_{1}, t_{*}\right)$ and that the spacetime cannot be extended so as to enlarge this interval. Suppose further that $H$ is positive somewhere and that the particle current density $N^{\alpha}$ does not vanish. Then $t=t_{*}$ is a curvature singularity in the sense that some curvature invariant blows up uniformly as $t \rightarrow t_{*}$.

Proof Note first that, as a consequence of condition (7) $H$ tends to infinity as $t \rightarrow t_{*}$. A spacetime with Kantowski-Sachs symmetry is, in particular, spherically symmetric. It is thus possible to define the area radius $r$ which, in terms of the coordinates used above, is just $B$. The gradient of $r$ is everywhere timelike and its length squared is $-\dot{B}^{2}$. The mass function $m$ is defined in a spherically symmetric spacetime by

$$
1-2 m / r=\nabla_{\alpha} r \nabla^{\alpha} r
$$

Thus in the Kantowski-Sachs case

$$
m=\frac{1}{2} B(1+\dot{B})^{2}
$$

In a spherically symmetric spacetime $m$ is monotone increasing along the integral curves of $\nabla^{a} r$ as long as the latter are timelike[1]. Hence $m$ can be bounded from below in terms of a positive constant only depending on the initial data. When studying spherically symmetric spacetimes it is useful to consider quantities defined on the quotient of spacetime by the group action. This space inherits a 2-dimensional metric $g_{a b}$ with Gaussian curvature $K$ and a 2-dimensional energy-momentum tensor $T_{a b}$. Let $\operatorname{tr} T=g^{a b} T_{a b}$. The Kretschmann scalar is given by

$$
\begin{aligned}
R^{\alpha \beta \gamma \delta} R_{\alpha \beta \gamma \delta} & =4 K^{2}+4 r^{-4}\left(\nabla^{a} r \nabla_{a} r\right)^{2}+r^{-4}\left(\frac{1}{2 r} \nabla^{a} r \nabla_{a} r-2 \pi r \operatorname{tr} \mathrm{T}\right)^{2} \\
& +16 \pi^{2} r^{-2}\left(T_{a b}-\frac{1}{2} \operatorname{trT} g_{a b}\right)\left(T^{a b}-\frac{1}{2} \operatorname{trT} g^{a b}\right)
\end{aligned}
$$

This formula also applies to spacetimes with plane symmetry and was used in [13] in that context. All terms except the last are manifestly non-negative. The last is also nonnegative provided the energy-momentum tensor is diagonalizable. For Kantowski-Sachs 
symmetry this is automatic. Hence the Kretschmann scalar can be bounded below by $16 m^{2} / r^{6}$. Since it is known that $m$ is bounded below by a positive constant it follows that if $\lim _{t \rightarrow t_{*}} r=0$ the Kretschmann scalar will blow up as $t \rightarrow t_{*}$. Thus to prove the theorem we can assume without loss of generality that $r$ does not tend to zero at the singularity. The scalar curvature $R$ of the $t=$ const. hypersurfaces is simply $B^{-2}$. Thus under the present assumptions $R$ is bounded above. Given this information (3.4) can be used as in the Bianchi case (when the type is not IX) to show that $\operatorname{det} g \rightarrow 0$ as $t \rightarrow t_{*}$. By the conservation law $N^{0}$ tends to infinity in this limit. Condition (5), applied to the normal vector, shows that $T^{00}$ tends to infinity in this limit. In the Kantowski-Sachs case $T^{00}$ is an eigenvalue of the energy-momentum tensor and so $T^{\alpha \beta} T_{\alpha \beta} \rightarrow \infty$ as $t \rightarrow t_{*}$. This completes the proof.

It may be seen as unsatisfactory that this argument leaves open the nature of the curvature singularity in any given case. All that the above proof shows is that either $R^{\alpha \beta \gamma \delta} R_{\alpha \beta \gamma \delta}$ or $G^{\alpha \beta} G_{\alpha \beta}$ blows up as the singularity is approached. It will now be shown with the help of arguments of Collins[5] that the second of these quantities always blows up. According to the above proof, if $B$ does not tend to zero as $t \rightarrow t_{*}$ then the statement is true. One of the field equations reads

$$
2 B \ddot{B}+1+\dot{B}^{2}=-T_{1}^{1} B^{2}
$$

If $B$ tends to zero as $t \rightarrow t_{*}$ then $\dot{B}$ must be negative somewhere. Equation (5.6) then implies that it must stay negative. It follows from (5.6) that $d / d t\left(B\left(1+\dot{B}^{2}\right)\right) \geq 0$. In fact this is just the monotone property of the mass function mentioned earlier. It follows that, fixing some time $t_{0}$ and letting $B_{0}=B\left(t_{0}\right)$ :

$$
\dot{B}(t)^{2} \geq-1+B\left(t_{0}\right)\left(1+\dot{B}\left(t_{0}\right)^{2}\right) / B(t)
$$

for any $t>t_{0}$. Since $B \rightarrow 0$ it follows that $\dot{B} \rightarrow \infty$. Hence in particular for any $\epsilon>0$ the inequality $\epsilon(\dot{B} / B)^{2} \geq 1 / B^{2}$ holds close to the singularity. The explicit form of the Hamiltonian constraint in this case is

$$
\frac{2 \dot{A} \dot{B}}{A B}+\frac{\dot{B}^{2}}{B^{2}}+\frac{1}{B^{2}}=8 \pi T^{00}
$$

Hence

$$
H=-\left(\frac{\dot{A}}{A}+\frac{2 \dot{B}}{B}\right)=\left(8 \pi T^{00}-\frac{1}{B^{2}}+\frac{3 \dot{B}^{2}}{B^{2}}\right) /\left(\frac{-2 \dot{B}}{B}\right)
$$

This implies an inequality of the form

$$
H \geq C|\dot{B} / B|
$$

In other words $-d / d t(\log \operatorname{det} g) \geq-C d / d t(\log B)$ or $d / d t\left(\operatorname{det} g / B^{C}\right) \leq 0$. It follows that $\operatorname{det} g$ tends to zero as $t \rightarrow t_{*}$. Hence $T^{00}$ is unbounded and the same is true of $G^{\alpha \beta} G_{\alpha \beta}$.

In order to be able to apply Theorem 5.1 to the matter models discussed in Section 4 , it is necessary to verify that condition (7) holds for these matter models in the case 
of Kantowski-Sachs symmetry. Note first that (7) follows from condition $\left(7^{\prime}\right)$ as in the Bianchi case. The proof is similar to that given for Bianchi symmetry at the beginning of Section 4. In the Kantowski-Sachs case it is not known a priori that the scalar curvature $R$ is bounded above. However the evolution equation (2.3) for the mean curvature shows that on an interval where $H$ is bounded the integral in time of $k_{i j} k^{i j}$ is bounded. This is enough to apply the argument of [12] to obtain the boundedness of the metric. This, together with the boundedness of $(\operatorname{det} g)^{-1}$, shows that $R$ is in fact bounded and from that point on the argument proceeds as before. In the case of collisionless matter condition (7) was verified in [12]. For a perfect fluid of mixture of perfect fluids $\left(7^{\prime}\right)$ can be checked as much as in the Bianchi case. The only difference is in the parametrization of the 4-velocity of each fluid. This necesarily lies in the $(t, x)$ plane and so can be parametrized by its component in the $x$-direction.

Acknowledgements I thank Piotr Chruściel for constructive criticism of a previous version of this paper.

\section{References}

[1] G. BURNETT. Incompleteness theorems for the spherically symmetric spacetimes. Phys. Rev. D43 (1991), 1143-1149.

[2] C. CERCIGNANI. The Boltzmann equation and its applications. (Springer, 1988).

[3] P. T. CHRUŚCIEL, A. D. RENDALL. Unpublished.

[4] A. A. COLEY, J. WAINWRIGHT. Qualitative analysis of two-fluid Bianchi cosmologies. Class. Quantum Grav. 9 (1992), 651-665.

[5] C. B. COLLINS. Global structure of the Kantowski-Sachs cosmological models. $J$. Math. Phys. 18 (1977), 2116-2124.

[6] C. B. COLLINS, G. F. R. ELLIS. Singularities in Bianchi cosmologies. Physics Reports 56 (1979), 65-105.

[7] J. EHLERS. Survey of general relativity. In Relativity, Astrophysics and Cosmology. (Reidel, 1973).

[8] R. GLASSEY, W. STRAUSS. Asymptotic stability of the relativistic Maxwellian. Publ. R.I.M.S. Kyoto Univ. 29 (1993), 301-347.

[9] P. HARTMAN. Ordinary differential equations. 2nd Ed. (Birkhäuser, 1982).

[10] S. W. HAWKING, G. F. R. ELLIS. The large scale structure of space-time. (Cambridge University Press, 1973).

[11] X.-F. LIN, R. WALD. Proof of the closed universe recollapse conjecture for general Bianchi type IX cosmologies. Phys. Rev. D41 (1990), 2444-2448.

[12] A. D. RENDALL. Cosmic censorship for some spatially homogeneous cosmological models. Ann. Phys. 233 (1994), 82-96.

[13] A. D. RENDALL. On the nature of singularities in plane symmetric scalar field cosmologies. Preprint gr-qc/9408001 (1994) .

[14] I. M. SINGER. Infinitesimally homogeneous spaces. Commun. Pure Appl. Math. 13 (1960), 685-697. 
[15] R. WALD. Asymptotic behaviour of homogeneous cosmological models in the presence of a positive cosmological constant. Phys. Rev. D28 (1983), 2118-2120.

[16] R. WALD. General Relativity. (University of Chicago Press, 1984). 